\title{
Drawing on the Doctor-Patient Relationship in e-Health Services
}

\author{
Rolf WYNN ${ }^{\mathrm{a}, 1}$ \\ ${ }^{a}$ UiT The Arctic University of Norway, Tromsø, Norway
}

\begin{abstract}
The doctor-patient relationship has been the cornerstone of medical practice. As e-health is coming to play an increasingly important role, it is necessary to consider how e-health can draw on, integrate and safeguard aspects of this relationship and thereby improve services and engage patients.
\end{abstract}

Keywords. E-health, doctor-patient relationship, engagement

\section{Introduction}

The doctor-patient relationship is the cornerstone of medical practice [1]. This relationship is sometimes, and especially within the field of counseling, referred to as 'the therapeutic alliance' [2].

During the two last decades, there has been a gradual and significant increase in the availability and use of different e-health services, including on the web, on apps, and on video $[3,4]$. In Norway, the Covid-19 pandemic has resulted in an increase in the use of e-health also in the public health services, as the need to push back against the pandemic has forced many health providers to reduce the availability of face-to-face services [5]. As e-health now moves into a period with an increased uptake, it becomes important to consider how to draw on, integrate and safeguard the elements of traditional face-to-face communication that have been such an important part of clinical medicine and the doctorpatient relationship, in order to further the uptake and success of e-health services.

In this paper, we discuss some central characteristics of the doctor-patient relationship and the advantages that might be gained by e-health providers by taking these into consideration.

\section{Methods}

Drawing on central concepts from doctor-patient interaction research, we present and discuss some clinically important phenomena that e-health providers may consider in optimizing their services.

\footnotetext{
${ }^{1}$ Corresponding Author, Rolf Wynn, Department of Clinical Medicine, Faculty of Health Sciences, UiT The Arctic University of Norway, N-9037 Tromsø, Norway; E-mail: rolf.wynn@uit.no.
} 


\section{Results and Discussion}

The relationship between the doctor and the patient is the cornerstone of traditional medical treatment. This relationship draws on the doctor's professionalism in terms of her knowledge and skills, but it also relies on other central elements including confidentiality and trust between the parties $[1,2]$. This relationship is a tool in itself that may help patients in their healing $[1,2,6]$. The lack of trust in technology and lacking technical skills have been two central factors in discouraging people's use of e-health [7].

While it may be difficult to isolate which elements that are most effective in the provider-patient relationship, one can hypothesize that e-health services that emulate characteristics of this relationship will be more effective in engaging and helping patients. Some e-health tools provide an opportunity to transfer central elements of the face-toface relationship to a different medium, such as video consultations. However, this may be more challenging in other applications such as apps and self-help online programs. Drawing on this line of reasoning, some techniques that might be used to enhance engagement with e-health services could be video recordings or audio recordings of providers, the possibility to interact synchronously or asynchronously with providers through chat or email, etc. In this respect, interacting with providers, albeit in writing only, would probably be preferable to services where the user only provides or receives information, without any interactional element [8,9]. Regardless of the technology, it will remain central that the services inspire trust and require few technological skills.

\section{Conclusions}

As e-health is becoming an increasingly important part of the public health services, it is necessary to consider how e-health can draw on and integrate aspects of the traditional doctor-patient relationship in order to improve the services and engage the patients.

\section{References}

[1] Wynn R. Provider-patient interaction. Norwegian Academic Press, Oslo, 1999.

[2] Littauer H, Sexton H, Wynn R. Qualities clients wish for in their therapists. Scand J Caring Sci 2005; 19:28-31.

[3] Wynn R, Oyeyemi SO, Budrionis A, Marco-Ruiz L, Yigzaw KY, Bellika JG. Electronic Health Use in a Representative Sample of 18,497 Respondents in Norway (The Seventh Tromsø Study - Part 1): Population-Based Questionnaire Study. JMIR Med Inform. 2020;8:e13106.

[4] Wynn R, Gabarron E, Johnsen JK, Traver V. Special Issue on E-Health Services. Int J Environ Res Public Health. 2020;17(8):2885.

[5] Wynn R. E-health in Norway before and during the initial phase of the Covid-19 pandemic. Stud Health Technol Inform. 2020;272:9-12.

[6] Bystad M, Bystad C, Wynn R. How can placebo effects best be applied in clinical practice? A Narrative Review. Psychol Res Behav Manag. 2015;8:41-5.

[7] Mata-Cervantes G, Clay CE, Baxter C. Maximising patient uptake and engagement with eHealth. NHS England, 2017.

[8] Del Hoyo JD, Nos P, Faubel R, et al. Adaptation of TECCU App Based on Patients' Perceptions for the Telemonitoring of Inflammatory Bowel Disease: A Qualitative Study Using Focus Groups. Int J Environ Res Public Health. 2020;17:1871.

[9] Löhr H, Wynn R, Rosenvinge J. E-therapy as an adjunct to face-to-face therapy in the treatment of patients suffering from chronic psychiatric disorders. J Inform Technol Healthcare. 2007;5:67-79. 